\title{
Severe edema of the eyes and lips as rare side effects of eye ointment for protection of eyes under general anesthesia -A case report-
}

\author{
Sang-Jin Park, and In-Seong Kim \\ Department of Anesthesiology and Pain Medicine, College of Medicine, Yeungnam University, Daegu, Korea
}

Patients undergoing non-ocular surgery under general anesthesia may suffer from ocular complications because of the loss of protective reflexes. Simple taping of the eyelids closed, the instillation of ointments into the conjunctival sac, and the use of protective goggles have been recommended for eye protection. We observed a case of a 6 -year-old child undergoing tonsillectomy and adenoidectomy who experienced severe edema after application of an ointment (Terramycin ophthalmic ointment) to the orbits and upper lip. After several days of steroid and antihistamine administration, the edema of the orbits and lip returned to normal. Therefore, we suggest that unexpected edema in the orbits and lip following surgery can be due to hypersensitivity to the ointment. (Korean J Anesthesiol 2012; 63: 454-456)

Key Words: Eye protection, Hypersensitivity, Ointment.

Bell's phenomenon, the blink reflex, and the eyelid reflex are normal protective mechanisms of the eyes. However, ocular injuries can occur during surgery because these reflexes disappear under general anesthesia. In particular, the possibility of ocular injuries increases during lumbar laminectomy [1], head and neck surgery, and surgery performed in the lateral decubitus position [2]. The most common ocular injury during surgery is corneal abrasion [2], and this leads to medical malpractice lawsuits more frequently than injuries of other body parts [3]. Thus, instillation of eye ointment and taping the eyelids closed are recommended to prevent possible ocular injuries during surgical procedures [4]. We report a case of severe edema of the eyes and lips after instillation of eye ointment in a 6 -year-old patient undergoing tonsillectomy, with review of previous studies.

\section{Case Report}

Tonsillectomy and adenoidectomy of a 6-year-old patient with severe snoring was scheduled. He had no specific personal

Received: October 20, 2011. Revised: 1st, November 22, 2011; 2nd, November 28, 2011; 3rd, December 1, 2011; 4th, December 7, 2011. Accepted: December 7, 2011.

Corresponding author: Sang-Jin Park, M.D., Department of Anesthesiology and Pain Medicine, College of Medicine, Yeungnam University, 317-1, Daemyung-dong, Nam-gu, Daegu 705-717, Korea. Tel: 82-53-620-3365, Fax: 82-53-626-5275, E-mail: apsj0718@naver.com

(C)This is an open-access article distributed under the terms of the Creative Commons Attribution Non-Commercial License (http:// creativecommons.org/licenses/by-nc/3.0/), which permits unrestricted non-commercial use, distribution, and reproduction in any medium, provided the original work is properly cited. 
or family history of asthma or allergies. Preoperative vital signs, blood tests, urinalysis, electrocardiogram, physical exam, and chest X-ray findings were all normal as well.

As premedication, $0.25 \mathrm{mg}$ of intramuscular atropine sulfate was injected. After the patient arrived in the operating room, electrocardiography, pulse oximetry, and noninvasive blood pressure monitoring were begun. Pre-anesthetic blood pressure was $130 / 80 \mathrm{mmHg}$, heart rate was 120 beats/min, and $\mathrm{SpO}_{2}$ was $99 \%$. Intravenous injection of thiopental sodium $125 \mathrm{mg}$ and recuronium bromide $20 \mathrm{mg}$ was performed for induction. During the maintenance phase of general anesthesia, 1.5 vol\% of sevoflurane, $1.5 \mathrm{~L} / \mathrm{min}$ of air, $1.5 \mathrm{~L} / \mathrm{min}$ of oxygen, and $0.5 \mu \mathrm{g}$ / $\mathrm{kg} / \mathrm{min}$ of remifentanil $\mathrm{HCl}$ were used. Endotracheal intubation was performed with no difficulty using a $5 \mathrm{~mm}$ diameter anode tube. After intubation, we applied oxytetracycline $\mathrm{HCl}$ / polymyxin B sulfate (Terramycin ophthalmic ointment ${ }^{\circledR}$, Pfizer, Jakarta, Indonesia) eye ointment to both eyes, and taped the eyelids closed with paper surgical tape. We applied the same ointment to the upper lip to prevent lip injuries due to the use of a Davis-Crowe mouth gag during tonsillectomy. Tonsillectomy

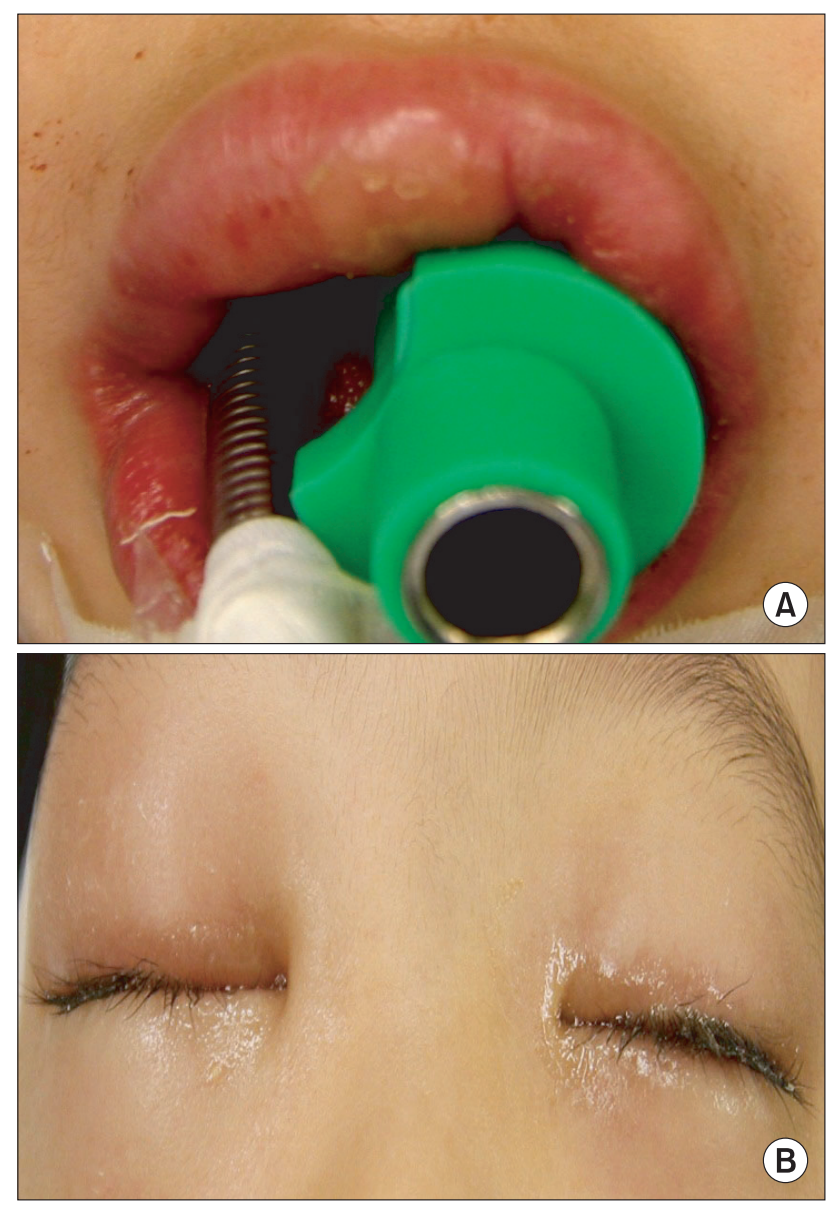

Fig. 1. The edematic change in the upper lip (A) and both eyelids (B). was performed with the head elevated 10 degrees and the neck extended. Vital sign were stable throughout the operation, and the surgery ended with no specific problems.

Removing the mouth gag, we found swelling of the patient's upper lip (Fig. 1A) and severe bilateral periorbital edema (Fig. 1B). Both conjunctivae were swollen and injected as well. There was no evidence of injury due to surgical procedures, and there was no edema or urticaria in any other body part. Vital signs were stable, and there was no wheezing or change in the inspiratory pressure. We assumed that the symptoms were due to an allergic reaction of unknown etiology and injected 2 mg of chlorpheniramine maleate and $2 \mathrm{mg}$ of dexamethasone disodium phosphate. Even though there was no swelling other than in the eyelids and upper lip, we could not completely rule out laryngeal edema and thus decided to extubate after confirmation. Fiberoptic laryngoscopic examination (Olympus LF-GP, Olympus Medical Systems Corp., Tokyo, Japan) showed no upper laryngeal or epiglottic edema. After suctioning the oral secretions, the cuff of the endotracheal tube was deflated and we could confirm an air leak around the tube. Re-intubation and tracheostomy sets were prepared in case of unexpected airway obstruction, and the endotracheal tube was removed after complete recovery of spontaneous respirations. After extubation, the patient's respirations were normal and his vital signs remained stable.

We consulted ophthalmologists and dermatologists, and these specialists concluded that, since we had seen follicular reaction and injection in the conjunctivae, with no corneal lesions or visual disturbances, the symptoms were due to an allergic reaction of the eyelids, conjunctivae, and lips, which would recover as time passed. We explained this to the patient's parents, and decided to treat him conservatively using steroid eye drops and intravenous steroids and antihistamines. The patient was moved to the general ward, and complained of no specific symptoms other than some itching sensations in the lips and periorbital area. On postoperative day 2 , the edema was almost completely relieved, and the patient was discharged. Follow-up physical examination after one week showed no edema at all.

\section{Discussion}

Instillation of eye ointment or taping of the eyelids closed is performed to prevent possible ocular injuries during general anesthesia $[4,5]$. The latter method is simple and effective, but there has been a case report of periorbital edema as an allergic reaction to the tape [6]. In the case discussed here, edema was present not only in the eyelids and conjunctivae, but also in the upper lip, to which the ointment had also been applied. We observed injection and follicular reaction in the conjunctivae 
as well. Accordingly, we assumed that the edema had occurred due to the ointment, rather than the tape.

Allergic reaction of the eyes due to contact with external allergens causes conjunctival injection, follicular conjunctivitis, periorbital edema, and pruritus [7]. The signs that the patient in our case showed are consistent with an allergic reaction, considering that he suffered from conjunctival injection, conjunctival follicles, and periorbital edema. Certainly, allergic reaction to the muscle relaxant, inhalation anesthetic, thiopental sodium, or remifentanil is to be considered. However, allergy related to the drugs frequently used during anesthesia is usually accompanied by systemic reactions such as hypotension, tachycardia, hypoxia, high airway resistance, and more extensive skin reactions [8-10]. Physical irritation during anesthetic induction or surgery can cause edema as well. However, in this case there was no need to forcefully compress the patient's face with a mask, because ventilation was well maintained during the induction phase, and all body parts other than the mouth were covered with surgical drapes. The operator mentioned that he had not compressed the patient's face during the operation. There is little chance that edema had occurred due to position or physical irritation, since the surgery was performed in a supine position with the head elevated 10 degrees.

In an allergic reaction, the mainstay of treatment is conservative management using antihistamines, mast-cell stabilizing drugs, and steroids, and it is important to find the causative agent to prevent recurrence [7]. In this case, our therapeutic methods appear to have been proper, since the use of steroids and antihistamines promptly relieved the patient's symptoms. Eye ointments may be effective for protection of the eyes during surgery because they remain for a long time after instillation. However, a study showed that there was no significant difference in protective effect between taping of the eyes after applying ointment and taping only [11]. Furthermore, eye ointments can cause postoperative foreign body sensation and visual blurring [5], as well as periorbital edema and conjunctival injection, which were present in our case. Thus, routine instillation of eye ointments during surgical procedures should be considered with caution, even though it is performed for protection of the eyes.

In the case discussed here, edema of the eye and upper lip, conjunctival injection, and follicular conjunctivitis occurred after tonsillectomy and adenoidectomy. This response is assumed to have occurred due to an allergic reaction to eye ointment applied for protection of the eyes and lips. Therefore, eye ointment for protection of the eyes under general anesthesia should be used carefully and with more strict criteria.

\section{References}

1. Cucchiara RF, Black S. Corneal abrasion during anesthesia and surgery. Anesthesiology 1988; 69: 978-9.

2. Roth S, Thisted RA, Erickson JP, Black S, Schreider BD. Eye injuries after nonocular surgery. A study of 60,965 anesthetics from 1988 to 1992. Anesthesiology 1996; 85: 1020-7.

3. Gild WM, Posner KL, Caplan RA, Cheney FW. Eye injuries associated with anesthesia. A closed claims analysis. Anesthesiology 1992; 76: 204-8.

4. Grover VK, Kumar KV, Sharma S, Sethi N, Grewal SP. Comparison of methods of eye protection under general anesthesia. Can J Anaesth 1998; 45: 575-7.

5. Stiffring PA, Poulton TJ. Prevention of ophthalmic complications during general anesthesia. Anesthesiology 1987; 66: 569-70.

6. Tobias JD, Jagannathan N, Sawardekar A, Bhalla T. An unusual cause of postoperative orbital edema in a child after general anesthesia. Saudi J Anaesth 2011; 5: 105-7.

7. Chigbu DI. The management of allergic eye diseases in primary eye care. Cont Lens Anterior Eye 2009; 32: 260-72.

8. Mertes PM, Tajima K, Regnier-Kimmoun MA, Lambert M, Iohom G, Gueant-Rodriguez RM, et al. Perioperative anaphylaxis. Med Clin North Am 2010; 94: 761-89.

9. Vellore AD, Drought VJ, Sherwood-Jones D, Tunnicliffe B, Moore VC, Robertson AS, et al. Occupational asthma and allergy to sevoflurane and isoflurane in anaesthetic staff. Allergy 2006; 61: 1485-6.

10. Perbet S, Salavert M, Amarger S, Constantin JM, D'Incan M, Bazin JE. Fluoroderma after exposure to sevoflurane. Br J Anaesth 2011; 107: 106-7.

11. Orlin SE, Kurata FK, Krupin T, Schneider M, Glendrange RR. Ocular lubricants and corneal injury during anesthesia. Anesth Analg 1989; 69: 384-5. 